\title{
Time domain aero-thermo-elastic instability of two-dimensional non-linear curved panels with the effect of in-plane load considered
}

\author{
Hamid Moosazadeh ${ }^{1}\left(\right.$ IDohammad M. Mohammadi $^{2}$
}

Received: 24 February 2020 / Accepted: 23 August 2020 / Published online: 15 September 2020

(c) Springer Nature Switzerland AG 2020

\begin{abstract}
This study presents aero-thermo-elastic Instability of two-dimensional Non-linear Curved Panels. Aero-thermo-elasticity plays an important role in the design and optimization of supersonic aircrafts. Furthermore, the transient and nonlinear effects of the thermal and aerodynamic environment encompassing a curved surface cannot be ignored. Accordingly, a homogenous curved plate with a high length-to-width ratio and simply-supported boundary conditions is assumed. The effect of large deflection is included in the equations through von Kármán non-linear strain-displacement relations. The thermal load is assumed to be a steady-state temperature non-uniform distribution. Structural properties such as modulus of elasticity and thermal expansion coefficient are assumed to be temperature-dependent. The novelty is incorporating first- and third-order piston theory for the non-linear curved panel flutter analysis under the effects of inplane and thermal loads. Hamilton's principle is used and partial differential equations are derived. The semi-analytical weighted residual method for the nonlinear curved panel is utilized. The fourth- and fifth-order Runge-Kutta iterative method are deployed to obtain the non-linear aero-thermo-mechanical deflections. Non-linear frequency analysis of cambered panel with the combined effects of aerodynamics, thermal and in-plane loads is investigated for the first time. The increase in panel curvature leads to a complicated behavior in the non-linear structural frequency variations. With increasing in-plane compressive load, complicated oscillating behavior is observed. More critical instability boundary for cambered panel is detected through the use of third-order piston theory. In addition, with an increase in panel curvature from 0 to 3, the panel displacement increases and for higher camber ratio, it decreases.
\end{abstract}

Keywords Panel flutter · In-plane load · Thermal effects · First-order piston theory · Third-order piston theory · 2D panel · Time domain

$\begin{array}{ll}\text { List of symbols } \\ h & \text { Panel thickness } \\ H & \text { Curvature height } \\ H / h & \text { Curvature changes } \\ w_{0} & \text { Out-of-plane displacement } \\ a & \text { Plate width } \\ \eta & \text { Tension or compression force coefficient } \\ D & \text { Plate stiffness } \\ E & \text { Elastic modulus } \\ v & \text { Poisson's ratio }\end{array}$

$\bowtie$ Hamid Moosazadeh, hamid.moosazadeh@modares.ac.ir; Mohammad M. Mohammadi, Mohammadi.mm@mut.ac.ir| ${ }^{1}$ Department of Aerospace Engineering, Tarbiat Modares University, Tehran, Iran. ${ }^{2}$ Fuel Cell Technology Research Laboratory, Malek Ashtar University of Technology, Tehran, Iran.

$\begin{array}{ll}\underline{R}_{x} & \text { Radii of curvature } \\ \Delta P_{a} & \text { Aerodynamic pressure } \\ P^{d} & \text { Unsteady aerodynamic force } \\ P^{s} & \text { Initial static aerodynamic force } \\ C_{\infty} & \text { Speed of sound } \\ U_{\infty} & \text { Free-stream steady velocity } \\ P_{\infty} & \text { Atmospheric pressure } \\ \rho_{\infty} & \text { Air density } \\ \gamma & \text { Isentropic gas constant } \\ M & \text { Mach number }\end{array}$

$\underline{R}_{x} \quad$ Radii of curvature

$p^{d} \quad$ Unsteady aerodynamic force 


$\begin{array}{ll}q_{\infty} & \text { Dynamic pressure } \\ \phi_{r} \xi & \text { Comparison functions } \\ U_{j,}, U_{i} & \text { Velocity vectors } \\ K & \text { Non-dimensional frequencies } \\ U & \text { Virtual strain energy } \\ V & \text { Virtual work } \\ K & \text { Virtual kinetic energy } \\ \vec{\sigma} & \text { Stress tensor } \\ \vec{\varepsilon} & \text { Strain tensor } \\ \overrightarrow{\mathbf{p}} & \text { External distributed force vector } \\ \overrightarrow{\mathbf{u}} & \text { Displacement vector } \\ \overrightarrow{\mathbf{u}}, t & \text { Velocity vector } \\ N_{x} & \text { In-plane axial force resultant } \\ M_{x} & \text { Bending moments resultant } \\ \delta_{e}, \delta_{\alpha} & \text { Modulus of elasticity and thermal expansion } \\ \Delta T & \text { Rise in plate temperature } \\ T & \text { Free stream temperature } \\ T_{* r e f} & \text { Reference temperature } \\ T & \text { Maximum in-plane temperature } \\ e_{T} & \text { Thermal variation coefficients for } \mathrm{E} \\ \alpha_{T} & \text { Thermal variation coefficients for } \alpha \\ \alpha & \quad \text { Thermal expansion coefficient } \\ C_{r} & \text { In-plane load coefficient } \\ R_{x} & \text { Magnitude of in-plane load } \\ \Omega_{0} & \text { First frequency } \\ \bar{\Omega} & \text { Non-dimensional frequency } \\ & \end{array}$

\section{Introduction}

Non-linear structural vibration with the effect of elasticity, inertial and aerodynamic interaction is a topic of aeroelasticity science. Panel dynamics is introduced with both dynamic flutter and static divergence due to in-plane compressive load. As a non-linear, non-conservative phenomenon, panel flutter displays a wide range of behaviors from the state of static stability to chaotic instability. The effect of heat gradient or predefined load caused by inappropriate attachment in joints leads to compressive load in panel boundaries. The effect of curvature or deformation in the flutter boundary and aeroelastic instability is of crucial importance. In the present paper, a new approach to consider different loads on panels is investigated. For a two-dimensional (2D) curved panel, the value of oscillation amplitude during flutter is nearly equal to the curvature magnitude. Due to the existence of curvature, aerodynamic loading affects the flutter boundaries. In comparison with theoretical results, the experimental results of curved panel flutter have demonstrated a suitable consistency.

The non-linear flat panel flutter has been investigated by the effect of different environmental loads in the following articles. Using linear structural theory, Jordan [1] investigated the movement of unstable panels in the critical dynamic pressure. Dowell [2] studied the theoretical and experimental panel flutter in Mach numbers ranging from 1 to 5. Dogondgi [3], through the use of linear aerodynamics and linear isotropic panel theory, performed a comprehensive analytical investigation on the panel flutter at high supersonic velocities. Kuo [4] used perturbation and harmonic balance theories for the analysis of non-linear panel flutter system. Dowell [5] studied and extended non-linear panel flutter. Dorci [6] performed an aeroelastic analysis including thermal and aerodynamic effects to design the re-entry launch vehicle (as an elastic system). He studied the capability of producing deformation, thermomechanical stresses and changes in structural properties which lead to unstable aeroelastic behavior. Guo and Mei [7] investigated the use of aeroelastic modes in non-linear panel flutter analysis in view of thermal effects. Culler [8] investigated the combination of fluid-heat-structure for non-linear aero-thermo-elastic analysis in supersonic flow. Perez [9] used the non-linear reduced order method for thermo-elastodynamic response analysis of isotropic panels and FG materials. Visbal [10] studied the interaction between the horizontal shock and a flexible panel.

Research in the field of curved panel flutter is also abundant. Fung [11] studied the static stability of a 2D curved panel for supersonic flutter phenomenon. Yates and Zeydel [12] studied the curved panel flutter using a linear analysis. Anderson [13] obtained experimental results for a supersonic curved panel. Steerman et al. [14] carried out experiments on cylindrical shell flutter. Bolotin [15] provided the equations of curved panel without any quantitative results. Houbolt [16] investigated certain aero-thermo-elastic problems related to aircraft structure at high velocities. Schaeffer et al. [17] analyzed the flat plate flutter under the effect of non-linear distributed heat loading. Dowell [18] studied the non-linear curved panel flutter using non-linear von Kármán relations and quasi-steady aerodynamic loading. Dowell and Venters [19] compared theoretical and experimental non-linear flutter of loaded plates. Yang [20] investigated buckled plate flutter through the use of finite element method (FEM). Xue et al. [21] studied the non-linear supersonic panel flutter using FEM with different temperature distributions. Bein et al. [22] analyzed the hypersonic curved panel flutter in view of the effect of aerodynamic heating. Zhou [23] employed FEM for the frequency domain modeling of non-linear flutter in composite plates. Libresco $[24,25]$ studied the vibration of geometrically-imperfect flat plate under thermal and mechanical loading as well as system frequency verses force in imperfect curved panels. Gee [26] investigated the non-linear panel flutter in combination with heat effect. Libresco [27] studied the linear and non-linear high temperature supersonic panel flutter. Yong and Shen [28] investigated the effect of geometric defect 
for FG plate. Abbas et al. [29] studied the parametric supersonic/hypersonic flutter and aero-thermo-elastic behavior of curved panels. Yang et al. [30] studied the flutter analysis of a hypersonic simply-supported curved panel using a two-way combination of aeroelasticity and aero-thermal effects. The non-linear geometric effect was taken into account based on the von Kármán model.

Ghoman and Azzouz [31] developed a FEM frequency domain procedure to predict the pre-flutter behavior and the flutter onset of curved panels simultaneously subject to aerodynamic and thermal load. Flutter coalescence frequencies and damping rates of the flutter of curved panel were investigated for 3D curved panels under increasing non-dimensional dynamic pressure and uniform temperature gradient load. The results of their study defined that the pre-flutter panel behavior and the flutter onset are altered when temperature loads are included. Shahverdi and Khalafi [32] presented a numerical analysis for the aero-thermo-elastic behavior of functionally-graded (FG) curved panels in hypersonic aerodynamic. To incorporate the applied aerodynamic pressure, the third-order piston theory was used. The generalized differential quadrature (GDQ) solution was implemented so as to discretize and solve the equations. They demonstrated the accuracy of the GDQ method for analyzing the aero-thermo-elastic behavior of FG curved panels. Recently, Amirzadegan and Dowell [33] studied the flutter and post-flutter LCO of elastic shells in a supersonic regime. They showed that the effects of streamwise and spanwise curvature are different, with the former lower the stability and the latter increase the stability.

In this study, we present the non-linear vibrational frequency analysis of curved panel under the effects of in-plane compressive and tensile loads is carried out for first time in time domain. Another novelty is incorporating first- and third-order piston theory for the non-linear curved panel flutter analysis under the effects of inplane and thermal loads. The effect of large deflection is included through von Kármán non-linear strain-displacement relations. Also, the effect of in-plane mechanical and thermal loads due to fluid flow viscosity over the panel is considered by assuming a temperature-dependent material. Additionally, boundary conditions are taken as simplysupported. The results are provided in two parts; in the first part, the non-linear frequency analysis of the curved panel structure along with the change in panel curvature and in-plane load effect is presented, and in the second part, non-linear aero-thermo-elastic analysis of the curved panel and panel behavior under the effect of different loads are investigated.

The present article includes introduction, equations, results, discussion, conclusion, nomenclature and references.
The introduction part includes a description of the research on flat plate flutter, curved plate flutter, panel flutter with the effect of thermal loads, experimental results of flutter, numerical methods of solving the panel flutter phenomenon, study of the effect of boundary conditions, etc.

The equations section includes a description of the theory and solution method. The results section includes graphs of vibration and aeroelastic analysis and verification with finite element method and with the use of other related articles. The discussion section explains the problem and the purpose and innovation and compares it with related articles and gives an overview of the results and their correctness.

\section{Formulation}

An infinitely-long cambered panel of width $a$, thickness $h$, maximum rise height $H$, and constant-radius cylindrical shell with curvature $\underline{R}_{x}$ is considered as in Fig. 1. The investigations in this paper mainly focus on the performances of 2D panels under fully aero-thermal-elastic interaction, which can be applied to the analysis and design of supersonic and hypersonic aircrafts. The effect of in-plane load $R_{x}$ and aerodynamic heating $T$ is also estimated.

The equations are derived taking into account the effect of aerodynamic heating with the use of virtual work. The virtual strain energy, virtual work done by applied forces and virtual kinetic energy are denoted by $\delta U, \delta V$ and $\delta K$ respectively [34].

$0=\int_{0}^{T}(\delta U+\delta V-\delta K) d t$

$\delta U=\int_{V} \overleftrightarrow{\sigma}: \delta \overleftrightarrow{\varepsilon} d V$

$$
\delta V=\int_{\Omega_{0}} \overrightarrow{\mathbf{p}} . \delta \overrightarrow{\mathbf{u}} d x d y
$$

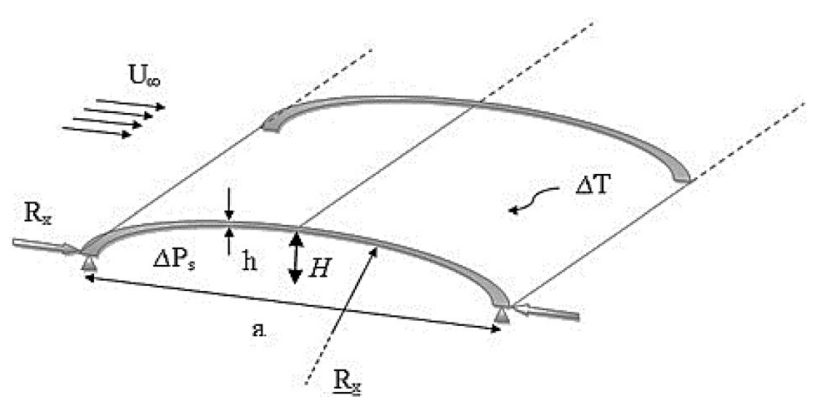

Fig. 1 Geometric model of 2D plate under the effect of external loads 
$\delta K=\int_{V} \rho \overrightarrow{\mathbf{u}}_{, t} \delta \overrightarrow{\mathbf{u}}_{, t} d V$

In the above equations, $\stackrel{\sigma}{\sigma}, \vec{\varepsilon}, \overrightarrow{\mathbf{p}}$, $\overrightarrow{\mathbf{u}}$ and $\overrightarrow{\mathbf{u}}_{t,}$ are denoted as stress tensor, strain tensor, external distributed force vector, displacement vector, and velocity vector, respectively, and $\Omega_{0}$ is defined as the mid plane. With the use of classic panel strain-displacement, Euler-Lagrange equations are defined. It is assumed that the structural equations are reduce from two dimensions to one dimension for infinite panel length. The structural bending equation is defined as $[29,30]$

$M_{x, x x}-N_{x}\left(w_{0, x x}-\frac{1}{R_{x}}\right)+\Delta P_{a}+\rho_{m} h w_{0, t t}=0$

where $w_{0}(x, t)$ is the plate's out of plane displacement, $N_{x}$ is the in-plane axial force resultant, $M_{x}$ is the bending and thermal moments resultant, $\Delta P_{a}$ is the aerodynamic pressure, and the final term of Eq. 3 is the panel transverse inertia and $\underline{R}_{x}$ is the radii of curvature. The $x$ direction is along $U_{\infty}$ and $\mathrm{t}$ is time domain solution.

Additionally, $M_{x} \equiv D w_{0, x x}$ and $D=E h^{3} / 12\left(1-v^{2}\right)$ where $D$ is the panel stiffness, $E$ is the elastic modulus, $v$ is the Poisson's ratio and $w_{0, x x}$ is the mid-plane curvature variation. The panel strain is defined via the non-linear von Kármán relation as $\varepsilon_{x}=u_{0, x}+1 / 2\left(w_{0, x}\right)^{2}+w_{0} / R_{1}[34]$.

The stress is generated on the panel boundaries due to the existence of supports. The axial stress, $N_{x}$, is the total in-plane load in the $x$ direction [30].

$N_{x}=N_{x}^{m}+N_{x}^{g}+N_{x}^{T}$

where $N_{x}^{m}$ is the mechanical tensile or compressive load, $N_{x}^{g}$ stems from curvature and non-linear terms, and $N_{x}^{T}$ is the in-plane thermal load, and are defined by

$N_{x}^{m}=\frac{a h}{\left(1-v^{2}\right)} \frac{1}{\int_{0}^{a} E(x)^{-1} d x} \eta$

$N_{x}^{g}=\frac{h}{\left(1-v^{2}\right)} \frac{1}{\int_{0}^{a} E(x)^{-1} d x}\left(\frac{1}{2} \int_{0}^{a}\left(w_{0, x}\right)^{2} d x+\frac{1}{R_{x}} \int_{0}^{a} w_{0} d x\right)$

$N_{x}^{T}=-\frac{1}{\left(1-v^{2}\right)} \frac{1}{\int_{0}^{a} E(x)^{-1} d x}\left((1+v) \int_{0}^{a} \alpha(x) \int_{-}^{h / 2} \Delta T d z d x\right)$

where $\eta$ is the tensile or compressive force coefficient per unit area on panel boundaries. $\Delta T$ is the rise in panel temperature with respect to the stress free state (reference temperature $T_{\text {ref }}$ ). In fact, a linear temperature distribution across the panel thickness is assumed as $\Delta T(x, z)=T-T_{\text {ref }}=T_{0}(x)+z T_{1}(x)[26,29]$.

At high speeds, the panel temperature rises to high values and reaches several hundreds of Celsius degrees. This leads to a reduction in flutter boundary and an increase in the LCO amplitude of the panel at the same dynamic pressure. The thermal effect is included in the panel equations for subtle panel flutter modeling.

The temperature distribution for high velocity flights is assumed to be in the steady state, and temperature variation along the thickness is disregarded. Hence, $\Delta T(x)=T_{0}(x)$ and, as a result, the in-plane thermal moment is neglected. The panel temperature is described by $T_{0}(x)=T \sin (\pi(x / a))$ where $T$ is the maximum in-plane temperature when $x=a / 2$. Simply-supported boundary conditions are defined as $w_{0}(x, t)=0$ and $w_{0, x x}(x, t)=0$ [35].

Material properties including elastic modulus $E$ and thermal expansion coefficient $\alpha$ are assumed to be temperature-dependent as in [29]

$$
\begin{array}{cr}
E=E_{0}+E_{1} T_{0}=E_{0}\left(1+e_{T} T_{0}\right), & e_{T}=E_{1 / E_{0}}<0 \\
\alpha=\alpha_{0}+\alpha_{1} T_{0}=\alpha_{0}\left(1+\alpha_{T} T_{0}\right), & \alpha_{T}=\alpha_{1 / \alpha_{0}}>0
\end{array}
$$

where $e_{T}$ and $\alpha_{T}$ are the thermal variation coefficients for $E$ and $\alpha$. Thermoelastic coefficients of the material depend on the position and temperature. Therefore $E=E(x, T)$ and $\alpha=\alpha(x, T)$.

\subsection{Aerodynamic loading}

Fluid-structure interaction is modeled based on the nonlinear piston theory. $\Delta P_{a}$ is the distributed pressure on the panel due to aerodynamic flow over the panel according to $\Delta P_{a}=P^{d}(x, t)+P^{S}(x)$ where $P^{d}(x, t)$ is the effect of unsteady aerodynamic force and $P^{s}(x)$ is the initial static aerodynamic force. Assuming an isentropic pressure on the panel and using the piston theory based on the downwash velocity $V_{z}$ in one dimension, one can write $[29,36]$

$P^{d}(x, t)=P_{\infty}\left(1+\frac{\gamma-1}{2} \cdot \frac{V_{z}}{c_{\infty}}\right)^{2 \gamma / \gamma-1}$

where $c_{\infty}$ is the sound speed and $\gamma$ is the isentropic gas constant. Based on a third-order expansion of Eq. 9, the third-order piston theory is derived as

$P^{d}(x, t)=P_{\infty}\left(1+\gamma \frac{M}{\beta_{1}}\left(\eta_{1} \frac{V_{z}}{c_{\infty}}\right)+\left[\frac{\gamma(\gamma+1)}{4}\right] \frac{M}{\beta_{1}}\left(\eta_{1} \frac{V_{z}}{c_{\infty}}\right)^{2}+\frac{\gamma(\gamma+1)}{12} \frac{M}{\beta_{1}}\left(\eta_{1} \frac{V_{z}}{c_{\infty}}\right)^{3}\right)$ 
where $\eta_{1}=M / \sqrt{M^{2}-1}$, and $c_{\infty}^{2}=\gamma P_{\infty} / \rho_{\infty}$ in which $P_{\infty}$ ， $\rho_{\infty}$ and $U_{\infty}$ are the atmospheric pressure, air density and free-stream steady velocity, respectively. Also, $\gamma=1.4$.

In order to define the aerodynamic pressure on the panel, the downwash velocity $\left(V_{z}\right)$ due to fluid flow over the panel is defined based on the panel vertical deflection as in $[18,29]$

$V_{z}=\left(\beta_{2} w_{0, t}+U_{\infty}\left(w_{0, x}+\hat{w}_{0, x}\right)\right)$

where $\hat{w}_{0, x}$ is the effect of initial imperfection or curvamethod for aeroelasti ture on the panel. The piston theory is a conventional $c$ analysis of a system in supersonic and hypersonic flows. The fluid flow only exists above the panel while the flow velocity below the panel is zero. For the Mach number $\mathrm{M}$, the dynamic pressure $q_{\infty}$ and constants $\beta_{1}$ and $\beta_{2}$, the following relations are taken into account: $M=U_{\infty} / c_{\infty}$ $q_{\infty}=\rho_{\infty} U_{\infty}^{2} / 2, \beta_{1}=\sqrt{M^{2}-1}, \beta_{2}=M^{2}-2 / M^{2}-1$. For high Mach numbers, $\beta_{1}=M$ and $\beta_{2}=1$.

\subsection{Non-linear aero-thermo-elastic equations of panel}

Non-dimensional system variables are defined according to
The effect of curvature is defined using [18]

$\frac{\hat{W}}{H}=\left[1-\frac{(x-a / 2)^{2}}{(a / 2)^{2}}\right]$

The above equation is transformed into $\hat{W}=-\left(\hat{h} / 2 \bar{h}^{2}\right) \xi(\xi-1)$.

\subsection{Galerkin method}

Galerkin method is implemented to solve the integro-differential equation (Eq. 13) so as to evaluate the structural response and the curvature impact on flutter boundary with thermoelastic properties. Moreover, simply-supported boundary conditions $\left(W=W_{, \xi \xi}=0, \quad \xi=0,1\right)$ are considered. The mode shape functions are defined such that the boundary conditions are satisfied:

$$
\begin{aligned}
W(\xi, \bar{t}) & =\sum_{i=1}^{n} a_{i}(\bar{t}) \phi_{i}(\xi) \\
\phi_{i}(\xi) & =\sin \left(\lambda_{i} \xi\right), \quad \lambda_{i}=i \pi
\end{aligned}
$$

Obviously, the approximate solution is not equal to the exact solution, and residual terms will remain. Multiplying

$$
\begin{aligned}
& W=\frac{w}{a}, \hat{W}=\frac{\hat{w}}{h}, \xi=\frac{x}{a}, \bar{t}=t \Omega_{0}, \Omega_{0}=\left(\frac{\pi}{a}\right)^{2} \sqrt{\frac{D_{0}}{\rho_{m} h}}, \bar{\Omega}=\Omega_{0} \frac{a}{c_{\infty}}, \bar{h}=\frac{h}{a}, \\
& \hat{h}=\frac{h}{\underline{\mathrm{R}}_{x}}, \bar{P}^{s}(x)=P^{s}(x) \frac{a^{4}}{D_{0} h}, T_{c r}=\frac{D_{0}}{E h a^{2} \alpha_{0}}, \bar{\rho}=\frac{\rho_{m}}{\rho_{\infty}}, H \approx \frac{a^{2}}{8 \underline{\mathrm{R}}_{\mathrm{x}}}, \tau=\frac{T}{T_{c r}}, \\
& \bar{T}=\tau \sin (\pi \xi), \mu=\frac{\rho_{\infty} a}{\rho_{m} h}, \beta=\sqrt{M^{2}-1}, \lambda=\frac{2 q a^{3}}{\beta D_{0}}, R_{x}=\frac{E_{0} h a^{2} \eta}{D_{0}\left(1-v^{2}\right)}=C_{r} \pi^{2}
\end{aligned}
$$

where $C_{r}$ is the in-plane load coefficient and $R_{x}$ is the magnitude of in-plane load. Using the non-dimensional quantities and substituting them in the above equations, the final non-linear aerothermo-elastic equation of the 2D panel is obtained as

$$
\begin{aligned}
(1 & \left.+\delta_{e} e_{T} T_{c r} \tau \sin (\pi \xi)\right) W_{, \xi \xi \xi \xi}-\left(1 /\left(\int_{0}^{1} \frac{d \xi}{1+\delta_{e} e_{T} T_{c r} * \sin (\pi \xi)}\right)\right) \\
& \times \frac{12}{\bar{h}^{2}}\left(\eta+\frac{1}{2} \int_{0}^{1}\left(W_{, \xi}\right)^{2} d \xi+\frac{\hat{h}}{\bar{h}} \int_{0}^{1} W d \xi\right)\left(W_{, \xi \xi}-\frac{\hat{h}}{\bar{h}}\right) \\
& -\left(1 /\left(\int_{0}^{1} \frac{d \xi}{1+\delta_{e} e_{T} T_{c r} \tau \sin (\pi \xi)}\right)\right)\left(\frac{1}{1-v} \int_{0}^{1}\left(1+\delta_{\alpha} \alpha_{T} T_{c r} \tau \sin (\pi \xi)\right) \tau \sin (\pi \xi) d \xi\right) \\
& \times\left(W_{, \xi \xi}-\frac{\hat{h}}{\bar{h}}\right)+\pi^{4} W_{, \bar{t} t}+\frac{M^{2} \pi^{4}}{\bar{h} \bar{\rho} \bar{\Omega}^{2} \beta_{1}} \eta_{1}\left(C_{a 1}\left(\beta_{2} \frac{\bar{\Omega}}{M} W_{, \bar{t}}+W_{, \xi}+\bar{h} \hat{W}_{, \xi}\right)\right. \\
& +C_{a 3} \frac{1+\gamma}{4} \eta_{1} M\left(\beta_{2} \frac{\bar{\Omega}}{M} W_{, \bar{t}}+W_{, \xi}+\bar{h} \hat{W}_{, \xi}\right)^{2}+C_{a 3} \frac{1+\gamma}{12} \eta_{1}^{2} M^{2} \\
& \left.\times\left(\beta_{2} \frac{\bar{\Omega}}{M} W_{, \bar{t}}+W_{, \xi}+\bar{h} \hat{W}_{, \xi}\right)^{3}\right)=\bar{P}^{s}
\end{aligned}
$$

the residual term or error by the proposed base function for the system mode shapes $\phi_{r}(\xi)=\sin (r \pi \xi), \quad r=1,2, \ldots, n$, integrating along the span and setting the result to zero, 
a series of ordinary differential equations are derived according to the number of expanded terms.

\section{Numerical results}

The equations derived in the previous section can be solved via numerical methods. To this aim, the fourth- and fifth-order Runge-Kutta model are used. A non-dimensional time range of up to 1000 is considered.

The results are presented in two sections. The first section concerns the non-linear frequency analysis of the panel structure with panel curvature changes and in-plane load effect. Non-linear aeroelastic analysis of the curved panel and its behavior under different loads are discussed in the second section. The number of extended modes is taken as 4, 6, and 8 which are then compared to each other and the correctness of results is evaluated through the increase of mode numbers. The six-mode solution is found to be a good choice.

The initial condition (IC) values for the non-linear panel vibration are assumed as $0.1,0.01$ and 0.001 such that with an increase in initial condition, the non-linear terms are excited even more. The analysis is performed for the aluminum panel with the conditions shown in Table 1.

\subsection{Non-linear frequency analysis of curved panel in time domain}

As the panel curvature changes from a flat state to a curved one, the pertinent structural frequency is plotted and compared for the linear and non-linear structure. Figure 2 shows the non-dimensional frequencies of the first and third mode with respect to camber ratio. The nondimensional non-linear frequencies with the small initial condition 0.0001 are the same as those of linear frequencies. It becomes clear that with increasing camber ratio, the first- and third-order non-linear frequencies rise, which is similar to what happened for linear frequencies.

Figure 3a shows the non-linear panel oscillation with the camber ratio of 5 and $I C=0.01$. With increasing panel camber ratio, the oscillation amplitude and asymmetry with respect to the equilibrium condition (around 0 ) increase. Non-linear frequency analysis of the curved panel structure under the effect of initial excitation of 0.01 is

Table 1 Plate properties

\begin{tabular}{lll}
\hline$\rho_{m}=2700 \mathrm{~kg} / \mathrm{m}^{3}$ & $a_{0}=5.762 \mathrm{e}-61 / \mathrm{k}$ & $a=1$ \\
$v=033$ & $a_{r}=6.074 \mathrm{e}-41 / \mathrm{k}$ & $h=0.01$ \\
$E_{o}=70 \mathrm{Gpa}$ & $e_{T}=-6.941 \mathrm{e}-41 / \mathrm{k}$ & $C_{-}=340 \mathrm{~m} / \mathrm{s}$ \\
$E_{a}=1.183 E_{o}=82.86 \mathrm{Gpa}$ & $\rho_{\infty}=1.225 \mathrm{~kg} / \mathrm{m}^{3}$ & $\gamma=1.4$ \\
\hline
\end{tabular}

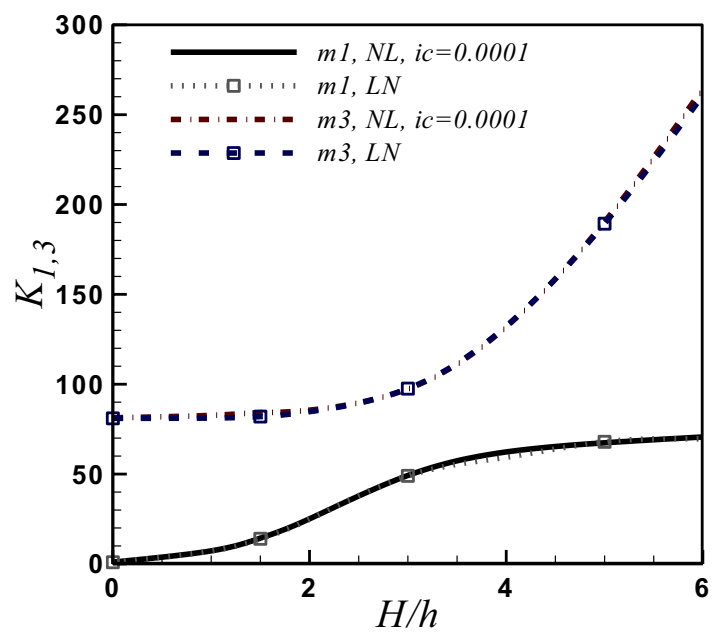

Fig. 2 Non-dimensional linear frequencies of curved plate for the first and second modes and IC $=0.0001$

performed in the time domain (Fig. 3b). Frequency values are indicated by $1.16,2.3$ and $3.63 \mathrm{~Hz}$.

Figure 4 a shows the first mode frequency of the nondimensional non-linear curved panel with respect to camber ratio for different initial conditions. As the value of initial condition increases up to 0.01 , the structural nonlinear terms are highlighted. With an increase in initial condition from 0.0001 to 0.002 , the frequency is reduced with increasing camber ratio. In contrast, as the initial condition increases to 0.01 , the frequency variation with respect to $H / h$ displays a more monotonous behavior. Figure $4 b$ shows that the third mode frequency is the same for initial conditions from 0.0001 to 0.002 and which increases with $H / h$. However, as the initial condition increases to 0.01 , the frequency suddenly rises while with increasing $H / h$, the frequency variation trend is reversed. This observation shows that the nonlinear analysis is highly dependent on initial conditions.

Figure 5 a shows the non-linear frequency variation with respect to in-plane load effect. According to the results, with decreasing in-plane load coefficient from 5 to 2.5 , the first mode frequency with $\mathrm{IC}=0.0001$ and 0.001 continuously decreases, while with decreasing $C_{r}$ from 2.5 to -10 , the frequency increases. For $\mathrm{IC}=0.003$, with decreasing $C_{r}$ from 5 to -4 , the frequency continuously decreases and then increases. In addition, with an increase of in-plane load after buckling (i.e. $C_{r}=-4$ ), the effect of non-linear terms increases and the non-linear frequency rises to 12 . With increasing initial condition to 0.01 , the variation of $C_{r}$ from 5 to -10 leads to a continuous reduction in frequency. Thus, the effect of initial condition on the nonlinear frequency variation and behavior with respect to in-plane load is emphasized. 


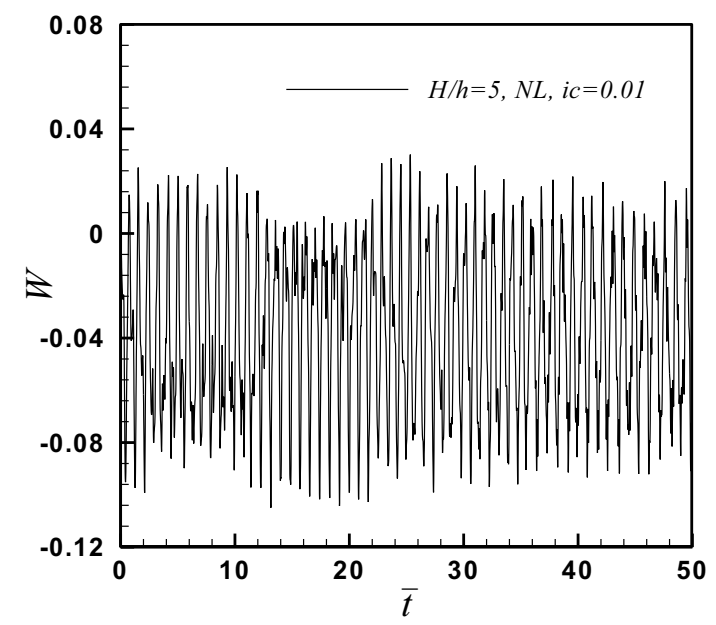

a $H / h=5$

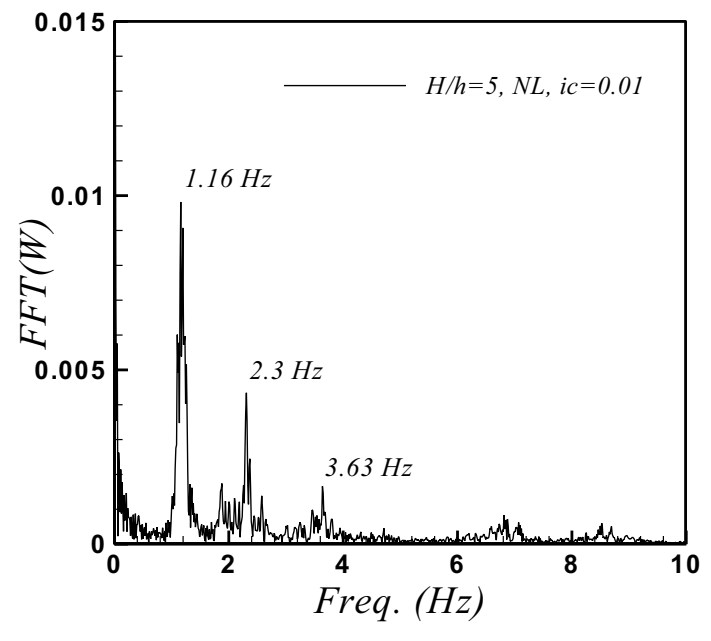

b $H / h=5$

Fig. 3 Non-dimensional displacement and frequencies of non-linear curved plate

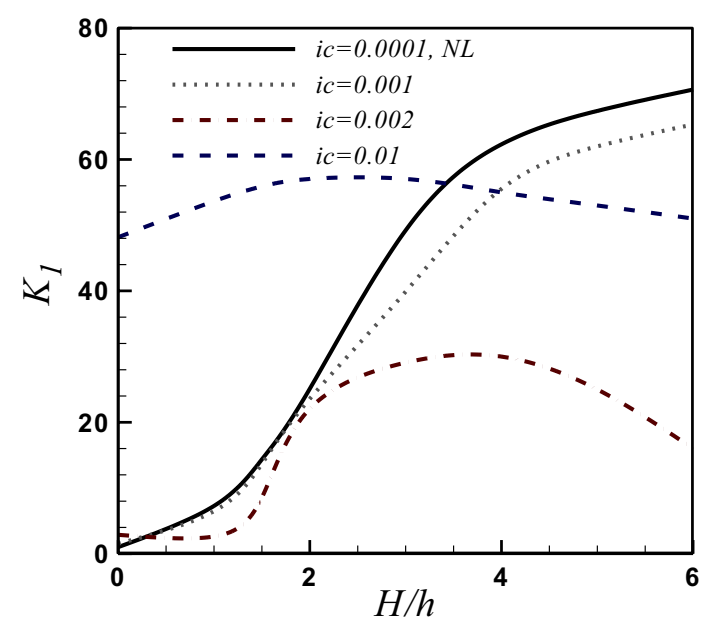

a First mode frequency

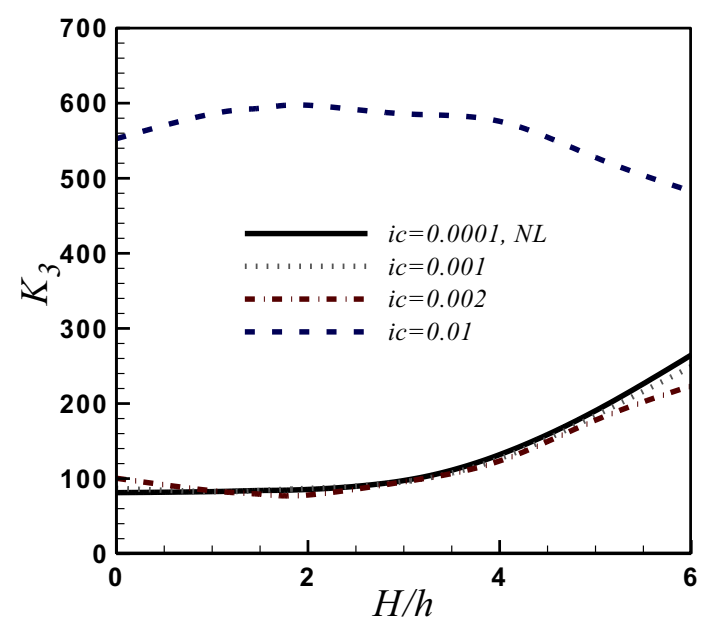

b third mode frequency

Fig. 4 Non-dimensional non-linear frequency of plate versus camber ratio

As the in-plane load coefficient decreases from 5 to -10 , the first three mode frequencies are continuously decreased for $H / h=1$ and $I C=0.01$. The results reveal that the effect of increasing the initial condition depends on the curvature ratio which brings about different behaviors. Hence, the curved panel is sensitive to the amount of inplane load and excitation condition.

\subsection{Non-linear aero-thermo-elastic analysis of curved panel}

In this section, the non-linear curved panel aeroelastic behavior with the effect of thermal and in-plane load being considered is investigated in time domain. 


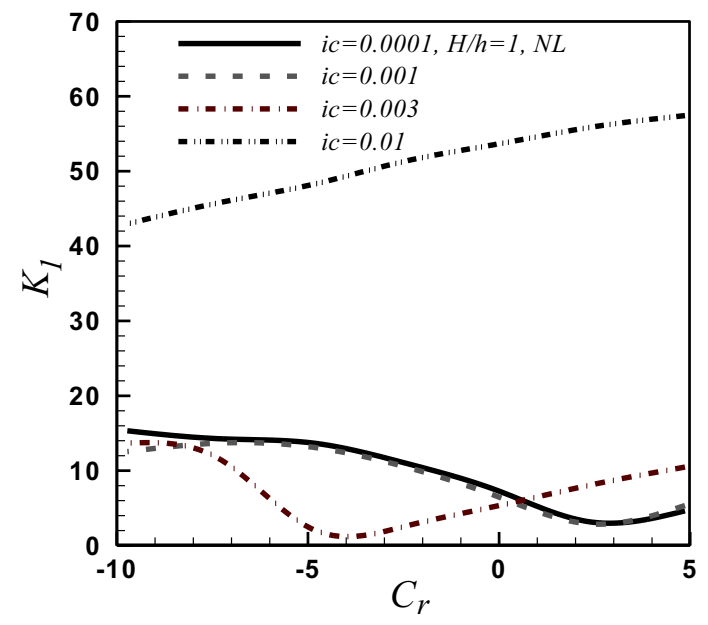

a variation of ic from 0.0001 to 0.01

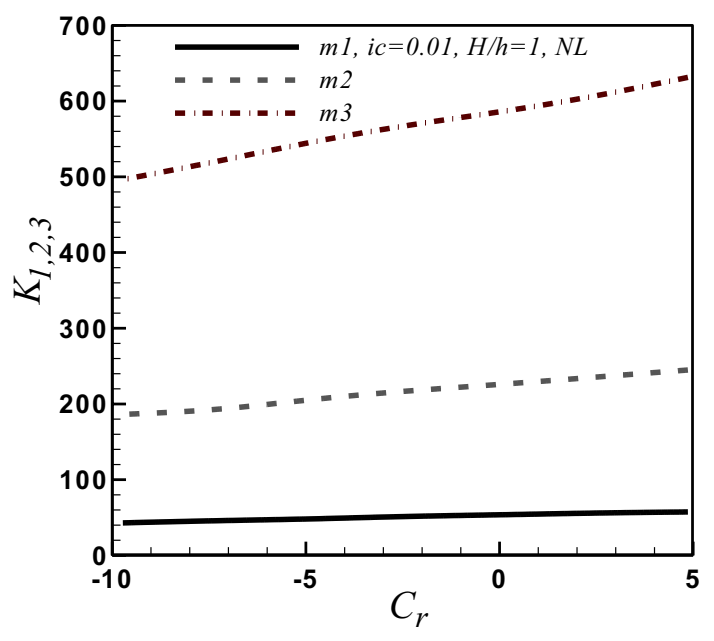

b first three mode frequencies

Fig. 5 Non-dimensional non-linear frequencies of plate $K$ for $H / h=1$ versus in-plane load

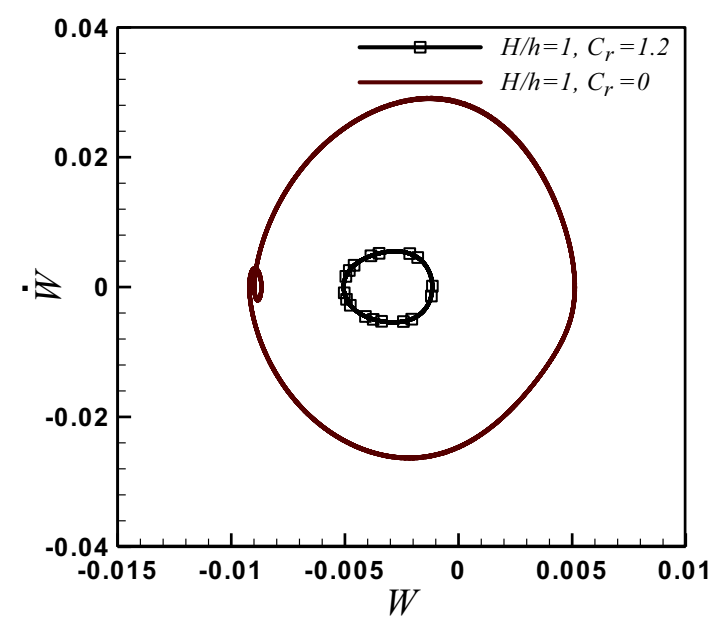

a- LCO, $C_{r}=0,1.2$

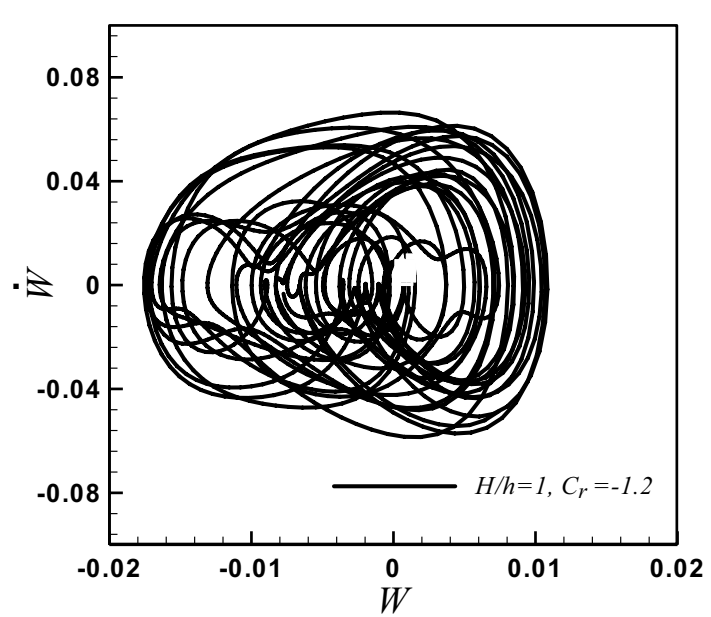

b- Chaotic, $C_{r}=-1.2$

Fig. 6 Limit cycle and chaotic motion of curved plate for $H / h=1$ in the presence of in-plane load $C_{r}$.

Figure 6a shows the LCO of the curved panel $(H / h=1)$ considering the effect of in-plane load. With a decrease in $C_{r}$ from 1.2 to 0 , the LCO changes from one-period to two-period motion and the increase in LCO bound can be distinguished. As the effect of compressive load increases, the panel behavior transforms from LCO into chaotic motion (Fig. 6b). One observes that the motion bound increases with the change of in-plane load from tension to compression.

In Fig. 7 a, the oscillation of the plane with curves 0 and 1 , are drawn, respectively. For both models, the panel behavior is LCO. But the shape of the LCO has changed with the curvature of the panel. Figure $7 \mathrm{~b}$ and $\mathrm{c}$ show the chaotic behavior of the panel with curves 2 and 3 , respectively. As the curvature of the panel increases, the amplitude of the oscillations increases. As the shell curvature increases to 4 and 5, the amplitude of the oscillations increases, but the behavior of the structure is converted into a fixed constant LCO band.

Figures $8 \mathrm{a}$ and $8 \mathrm{~b}$ show the harmonic LCO and chaotic motion of curved panel $(H / h=1)$ for the tensile and compressive load, respectively.

Figure 9a shows the non-linear flutter frequency $\left(K_{f}\right)$ of curved panel versus in-plane load at constant non-dimensional dynamic pressure $(\lambda=275)$. As $C_{r}$ decreases from 1.2 to -7.5 , the flutter frequency decreases. However, with a 

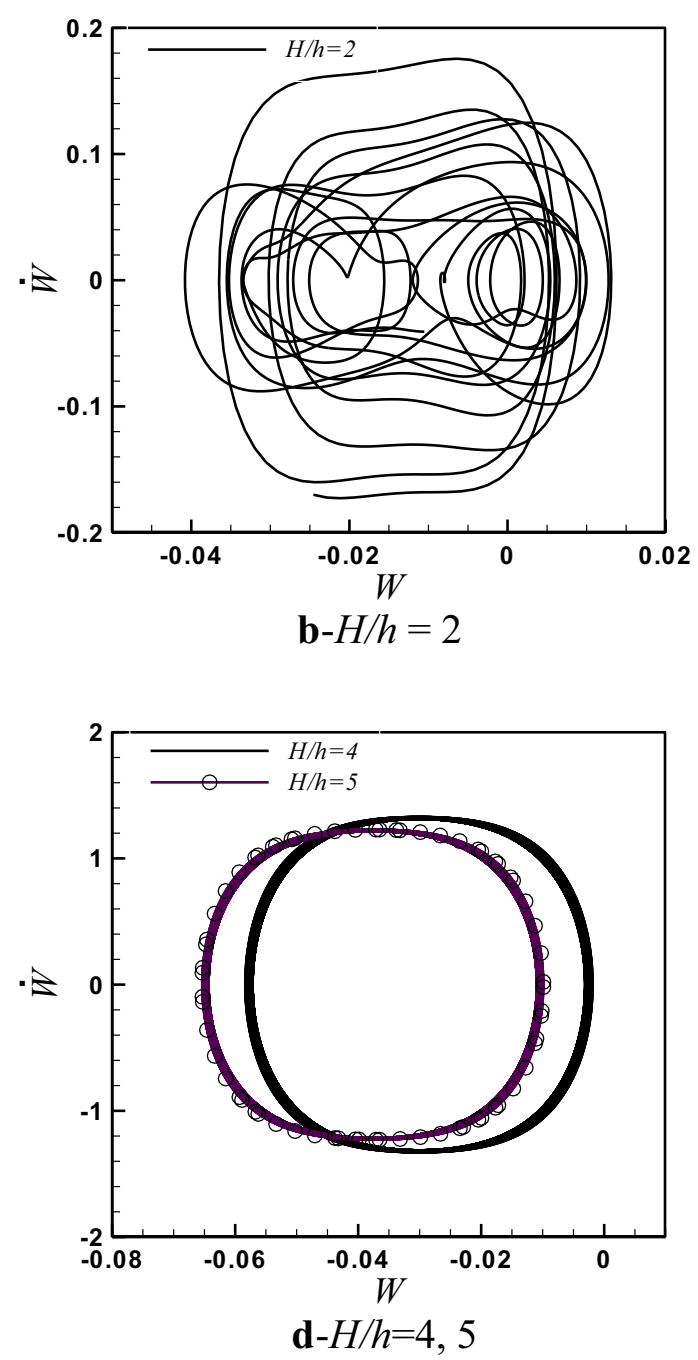

Fig. 7 LCO and chaotic motion of curved panel for $0<H / h<5$

decrease in $C_{r}$ from 1.2 to $-2, K_{f}$ decreases rapidly and LCO transforms into chaotic motion, with more decrease in $C_{r}$, $K_{f}$ decreases slightly decreases again. Figure $9 \mathrm{~b}$ shows the flutter dynamic pressure $\left(\lambda_{f}\right)$ versus $C_{r}$ for a camber ratio of 1 . As $C_{r}$ decreases from 1.2 to $-7.5, \lambda_{f}$ decreases rapidly. According to these analyses, it appears that the flutter frequency and flutter dynamic pressure of panel significantly vary in accordance to the in-plane load.

Figure 10a, b show the LCO of flat $(H / h=0)$ and curved panels $(H / h=1)$ for the thermal load coefficients $\tau=5$ ${ }_{,} \delta_{e}=1, \delta_{\alpha}=1$ through the use of first-order piston theory (PTA1) and third-order piston theory (PTA3), respectively. The panel behavior is LCO in both figures. In Fig. 10a, with increasing thermal load on the flat panel, the LCO motion change from a simple single-period to a double-period motion and the LCO boundary is extended. Similar results are obtained for the curved panel with $H / h=1$. The LCO motion of PTA1 is similar to that of PTA3 in Fig. $10 \mathrm{~b}$.

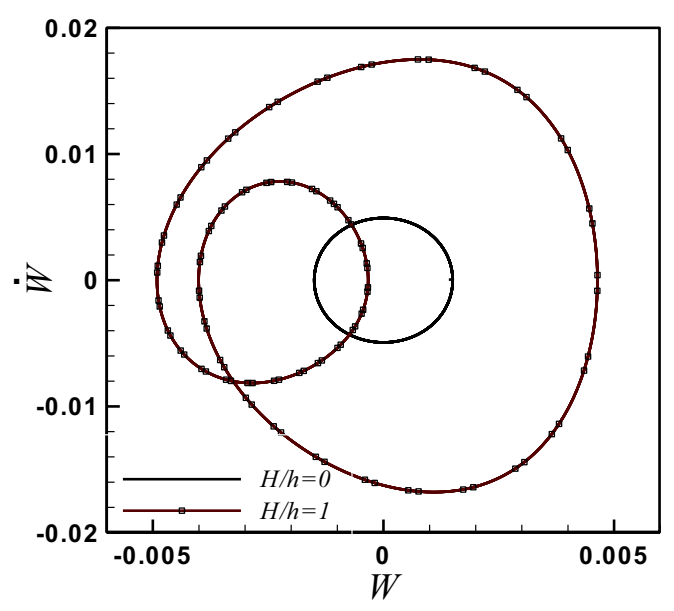

$\mathbf{a}-H / h=0,1$

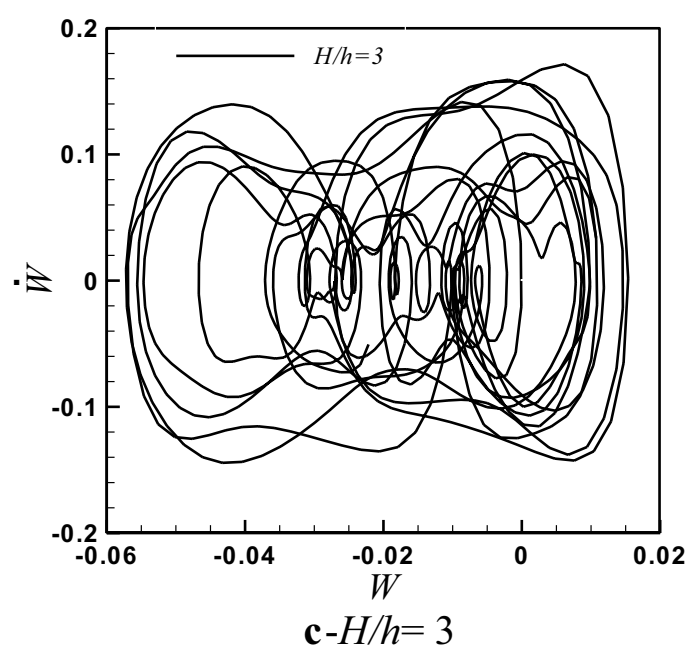

With increasing thermal load, the LCO motion grows and becomes complicated. The same behavior is shown with the use of PTA 1 and PTA3, although the LCO domain slightly shrinks.

Figure 11a shows the panel flutter dynamic pressure versus panel camber ratio obtained using PTA1 and PTA3. With increasing panel curvature, $\lambda_{f}$ is reduced. With the use of PTA1, $\lambda_{f}$ decreases from 350 (flat panel) to 80 (curved panel with the camber ratio of 5). This clearly emphasizes that PTA3 reduces the flutter dynamic pressure. This reduction is more highlighted for the flat panel at higher dynamic pressures. With increasing panel camber ratio, $\lambda_{f}$ decreases, and the dynamic pressure difference between PTA1 and PTA3 decreases and vanishes.

Figure $11 \mathrm{~b}$ shows the negative and positive oscillation amplitudes of the curved panel versus panel curvature with the use of PTA 1 and PTA3. With increasing panel curvature from 0 to 3 , the positive and negative amplitude 


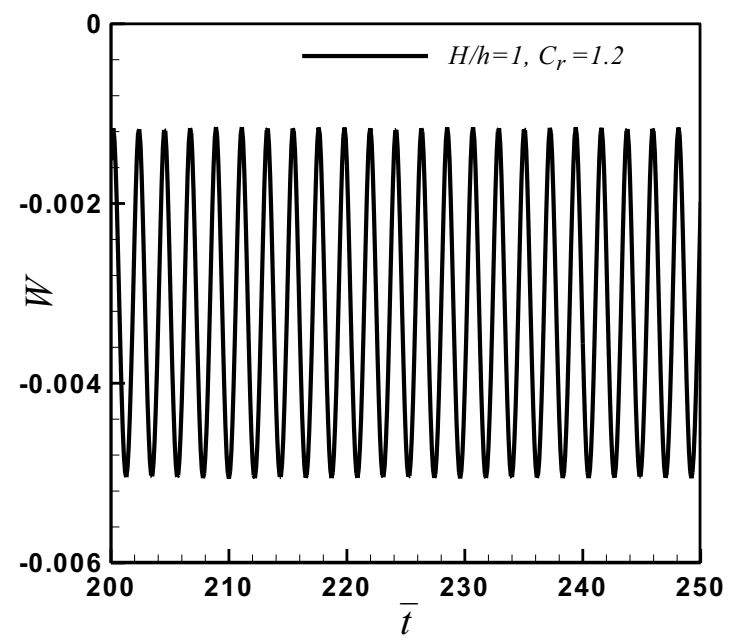

a- tension load, $C_{r}=1.2$

Fig. 8 Curved plate motion versus time

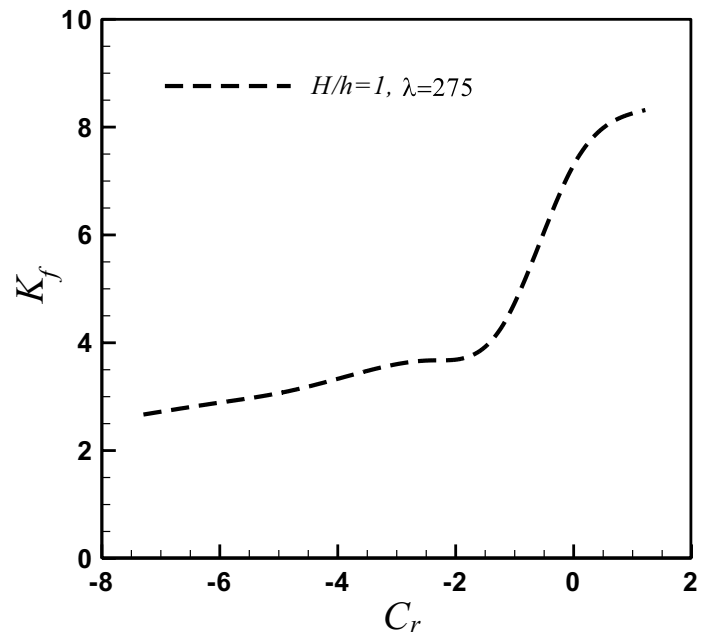

a - Flutter frequency

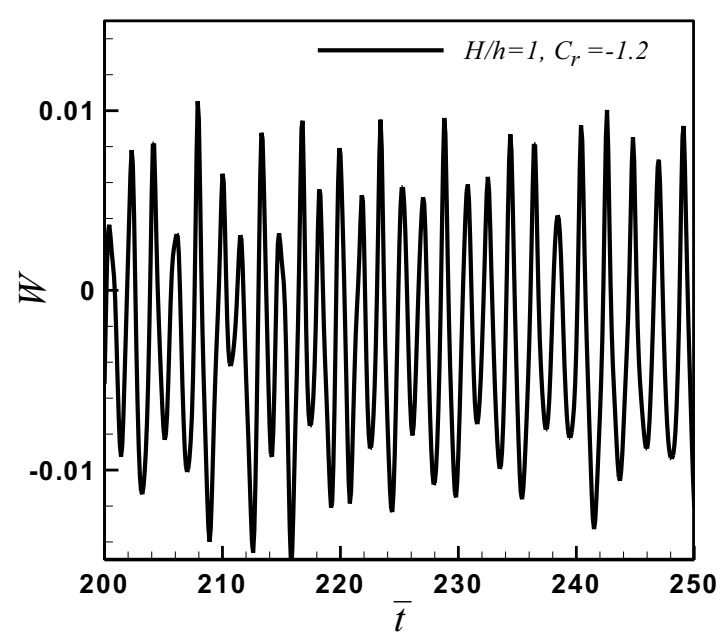

b- compression load, $C_{r}=-1.2$

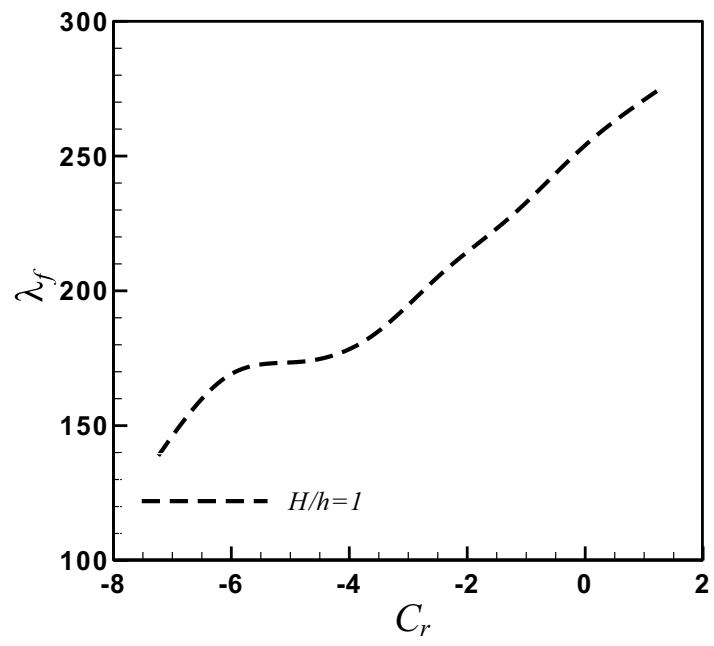

b - Flutter dynamic pressure

Fig. 9 Curved plate flutter frequency $K_{f}$ for $H / h=1$, and non-dimensional dynamic pressure $\lambda_{f}$

of oscillation increase, then decrease up to the camber ratio of 5 for the positive amplitude and reach a negative value. The LCO bound has the highest value for the camber ratio of 3. PTA3 shows a constant LCO bound for camber ratios in the range $2-5$ and the equilibrium point of $L C O$ increases as the camber ratio grows. The LCO bound is estimated to be higher for lower camber ratios based on PTA3 while being larger for higher camber ratios using PTA1.

Figure 12a shows the panel flutter dynamic pressure versus panel camber ratio found using PTA1 and PTA3. Simulations are conducted for $\tau=5, \delta_{e}=1, \delta_{\alpha}=1$ in this case. With increasing panel camber ratio to 1 for PTA $1, \lambda_{f}$ is reduced from 300 (flat panel) to 180 , then increase to 230 for a camber ratio of 1.5 . As the camber ratio increases from 1.5 to $5, \lambda_{f}$ decreases to 70 . This clearly shows that PTA3 reduces the flutter dynamic pressure although the trend of flutter dynamic pressure variation is the same. One concludes that the thermal load decreases $\lambda_{f}$ with respect to camber ratio.

Figure $12 \mathrm{~b}$ shows the negative and positive oscillation amplitude of the curved panel versus the panel curvature for $\tau=5, \delta_{e}=1, \delta_{\alpha}=1$ with the use of PTA1 and PTA3. With increasing panel curvature from 0 to 1 , the positive and negative amplitude of oscillation decrease, and with 


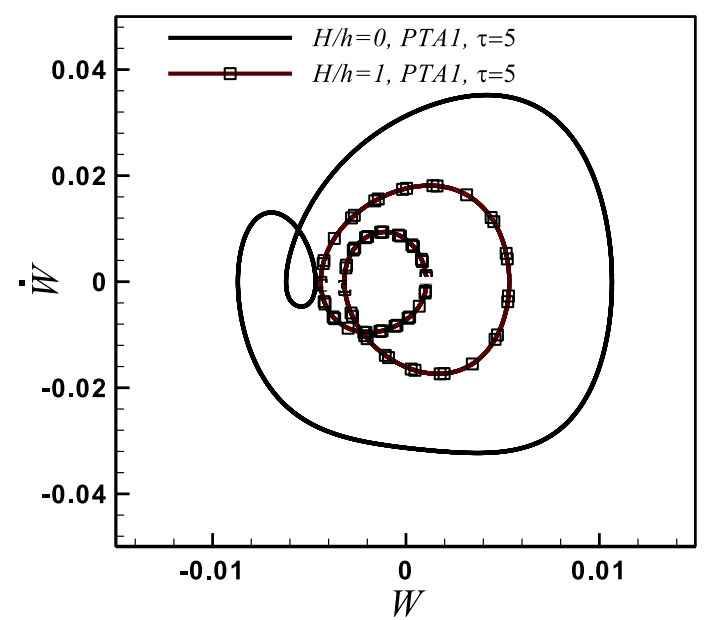

a - PTA1, $\tau=5$

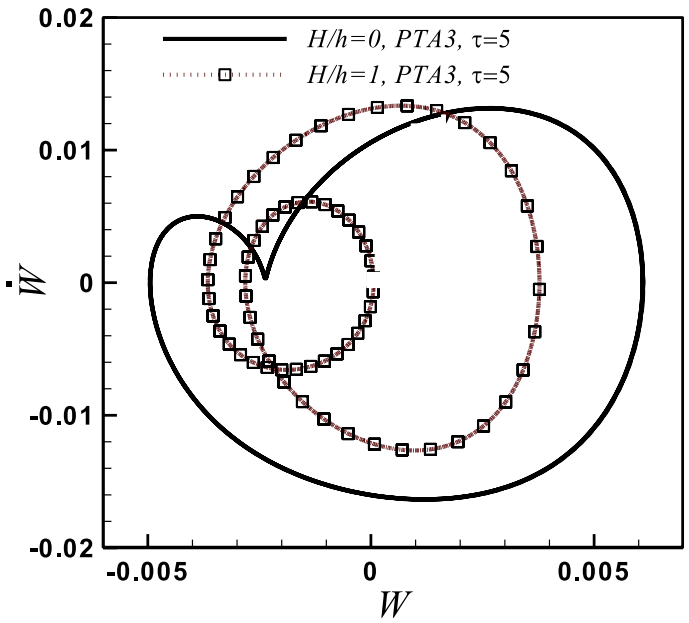

b $-\mathrm{PTA} 3, \tau=5$

Fig. 10 Limit cycle oscillation of curved and flat plates in view of thermal effects

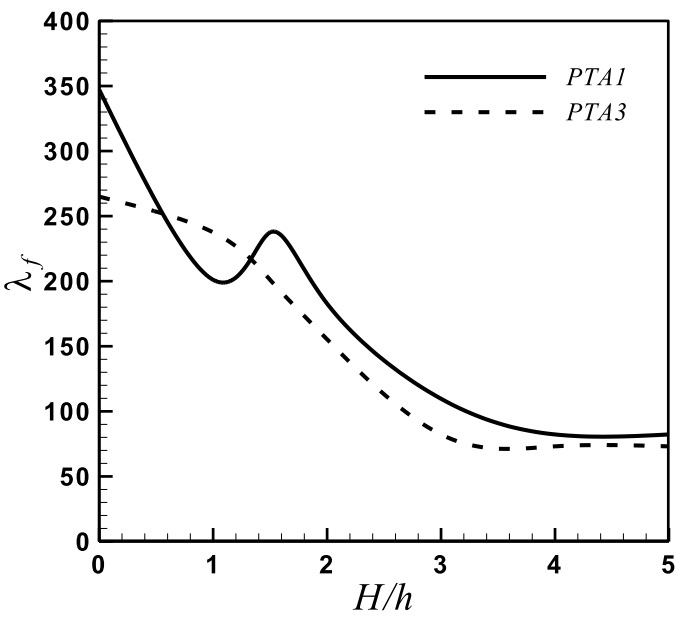

a - Dynamic pressure, $\lambda_{f}$

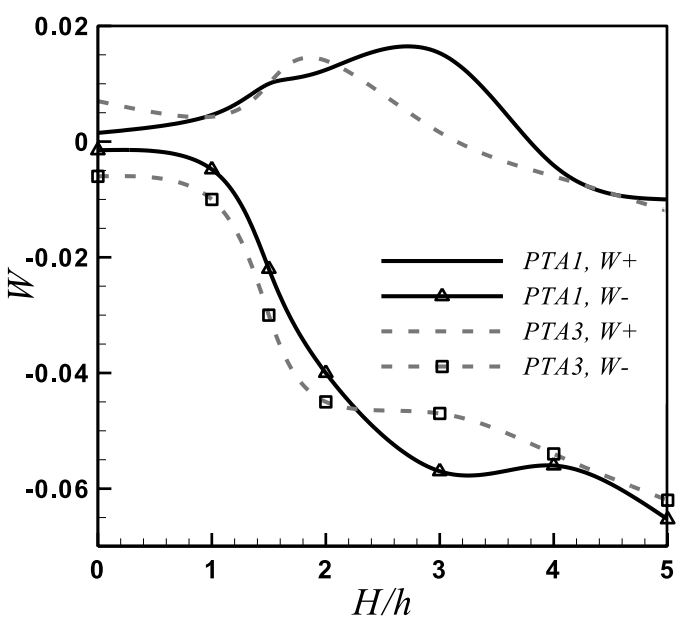

b - Displacement, $\mathrm{W}$

Fig. 11 Comparison of plate flutter dynamic pressure and oscillation amplitude versus curvature for PTA1 and PTA3

increasing panel curvature from 1 to 3 , these amplitudes decrease up to the camber ratio of 5 for the positive amplitude and reach a negative value. The LCO bound is maximum for the camber ratio of 3. The LCO amplitudes based on PTA1 and PTA3 are slightly different.

Figure 13a shows the panel flutter dynamic pressure versus panel camber ratio found using PTA 1 and PTA3. Simulations for this case are conducted for the in-plane load effect $\left(C_{r}=-2.43\right)$. With increasing panel camber ratio up to $1, \lambda_{f}$ of PTA 1 increases from 160 to 230 , and with increasing panel camber ratio from 1 to $5, \lambda_{f}$ decreases to 70. It is evident that, with the use of PTA3 instead of PTA1, the flutter dynamic pressure decreases. On the contrary, with increasing panel camber ratio from 0 to 5 , the difference between PTA1 and PTA3 diminishes. By considering the effect of mechanical in-plane load as in Fig. 13a, the variation of flutter dynamic pressure with respect to panel camber ratio changes beside Figs. $11 \mathrm{a}$ and $12 \mathrm{a}$ with and without thermal effects.

Figure $13 \mathrm{~b}$ shows the negative and positive oscillation amplitude of the curved panel versus panel curvature for $C_{r}=-2.43$ using PTA 1 and PTA3. With increasing panel curvature from 0 to 3 , the positive and negative amplitude of oscillation increase, then decrease up to the camber ratio of 5 for the positive amplitude and reach a negative value. The LCO bound is maximum for the camber 


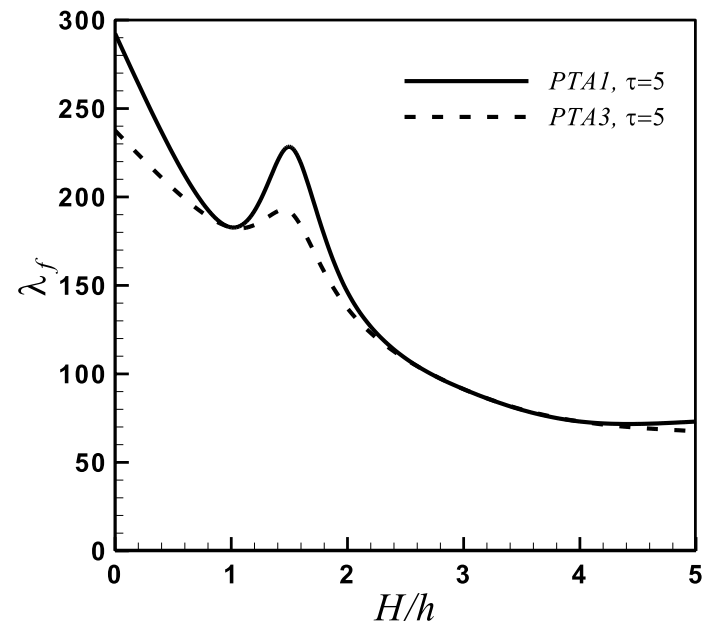

a - Dynamic pressure, $\lambda_{f}$

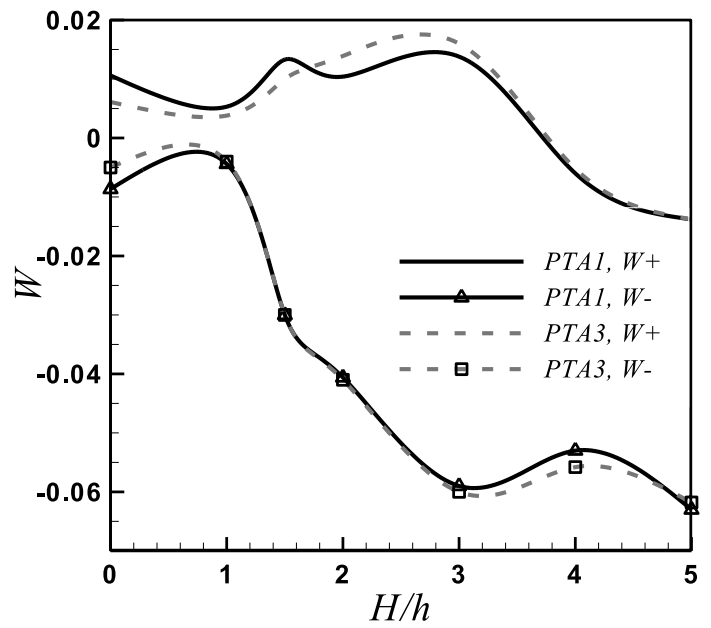

b - Displacement, $\mathrm{W}$

Fig. 12 Comparison of plate flutter dynamic pressure and oscillation amplitude versus curvature for PTA1 and PTA3 by considering the thermal effect $\tau=5$ and $\left(\delta_{e}=1, \delta_{\alpha}=1\right)$

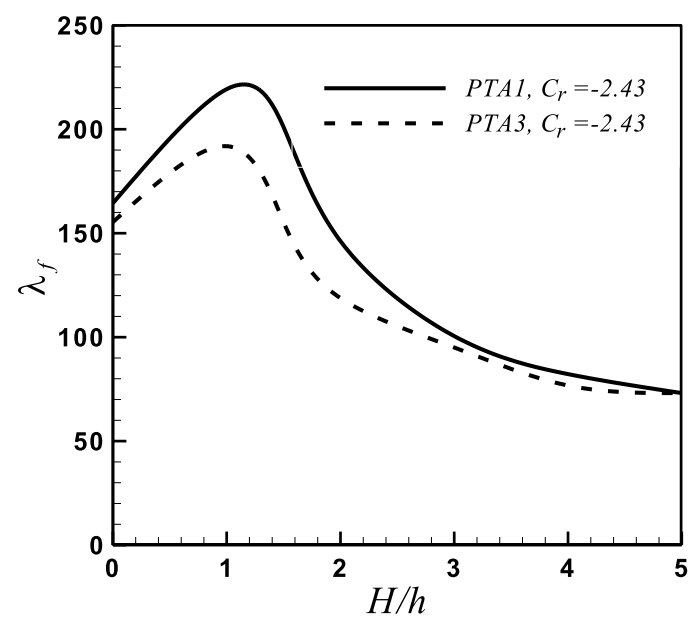

a - Dynamic pressure, $\lambda_{f}$

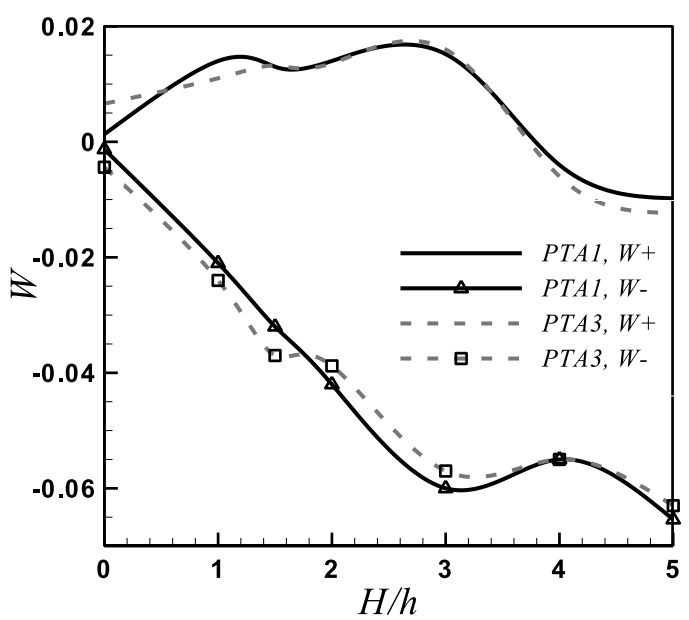

b - Displacement, W

Fig. 13 Comparison of plate flutter dynamic pressure and oscillation amplitude versus curvature for PTA1 and PTA3 by considering the effect of in-plane load $C_{r}=-2.43$

ratio of 3. The LCO amplitudes of PTA1 and PTA3 are also slightly different. As noticed, negative amplitude of oscillation increases linearly from the camber ratio of 0 to 3 . It is clear that the dynamic displacement amplitude of panel is affected by thermal and in-plane loads.

Figure 14 highlights the influence of temperature in conjunction with thermal degradation of the thermomechanical properties of panel material on the flutter dynamic pressure. With increasing panel curvature, the temperature dependency of structural properties leads to a reduction in the instability of dynamic pressure. For a camber ratio of 2 , the maximum difference between $\lambda_{f}$ of thermal-dependent and thermal-independent materials is shown.

\subsection{Verification}

The analysis of nonlinear vibrations of the structure using general FEM code is performed in this section and compared with the semi-analysis Gallerkin method. Two kinds of transient analysis and free vibration analysis are assumed for numerical solution of FEM. 


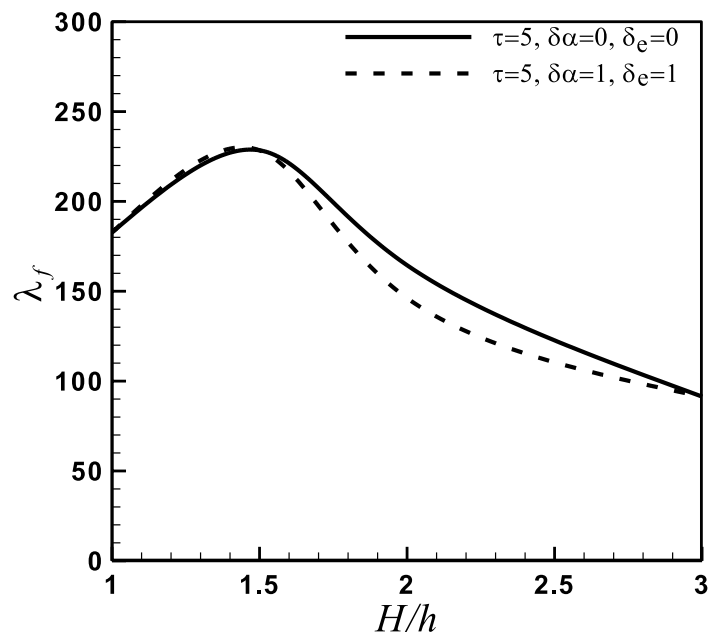

Fig. 14 Comparison between the effect of material temperaturedependency $\left(\delta_{e}, \delta_{\alpha}\right)$ in the flutter dynamic pressure $\lambda_{f}$

Figure $15 \mathrm{a}, \mathrm{b}$ show the comparative behavior of nonlinear curved panel oscillations, with curves 1 and 4, respectively, using the nonlinear FEM and the Gallerkin semi-analytical method. Figure 15 a shows the regular oscillating behavior of the panel for curvature 1, which shows a very good correlation between analytical and numerical results. In Fig. 15b, the oscillation of the panel is examined with a curvature of 4 , which shows a very good correlation between the chaotic vibrational behaviors in both methods.

Figure 16a compares the changes in the first and third linear frequencies of the curved panel with two numerical and semi-analytical method as FEM and Gallerkin, which are very close to different curves. Numerical analysis in this section is done in two part, the structural linear modal analysis and the transient dynamic analysis for small initial stimulation. Both analysis lead to the same answer. In Fig. 16b, the first and second nonlinear frequencies of the curved panel are compared, in terms of curvature, by the FEM and Gallerkin method. The first frequency is matched very good and the second one is slightly different. In general, in nonlinear analysis with large structural excitation, the frequencies have shown low changes in terms of curvature change.

The present study compares the activities of Dowell, Abbas, Anderson and Epureanu. This comparison is given below.

The curved panel displacement amplitude versus nondimensional flutter dynamic pressure for different curvatures is plotted in Fig. 17a. With an increase in dynamic pressure and panel camber ratio, the displacement amplitude increases. The non-dimensional displacement is defined as $W=w / h$. The results are compatible with the Dowell's solution [18].

The curved panel flutter Mach number versus thermal load effects for temperature-dependent properties $(\delta \alpha=1, \delta e=1)$ and temperature independent properties $(\delta \alpha=0, \delta e=0)$ is compared with the solution of Abbas [29] in Fig. 17b. With increasing thermal load coefficient, the system flutter Mach number decreases and reaches 3 for $\tau=10$ from 6.6 for $\tau=0$.

For the nonlinear flat panel with the effect of the inplane load $\left(C_{r}=-2.8\right)$, the present study has been compared with the Epureanu research [37]. The limited cycle diagram is shown in Fig. 18a. The results are fully consistent in both try.

Experimental study of three-dimensional panel flutter with fixed boundary conditions has been performed by Andersen. For the two-dimensional curved plane, the
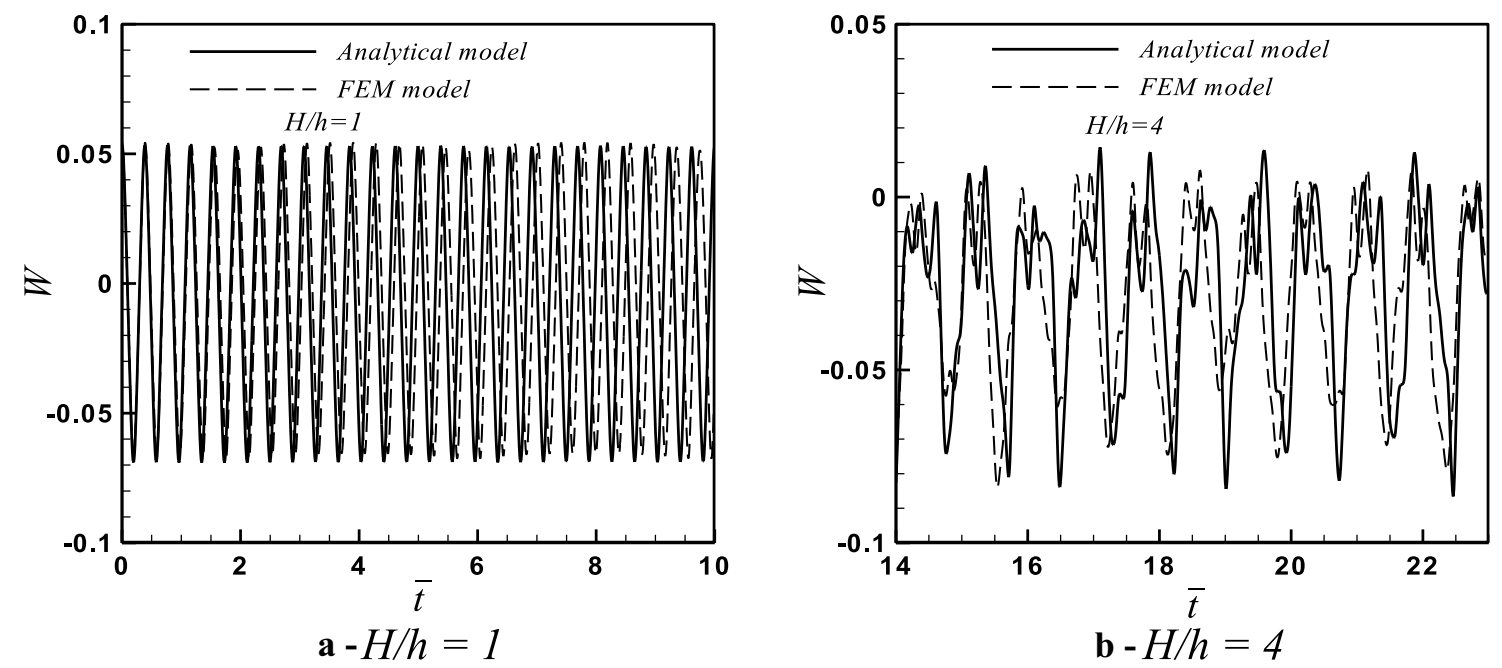

Fig. 15 Comparison of the scope of curved panel oscillation with different curves by FEM and Gallerkin method 


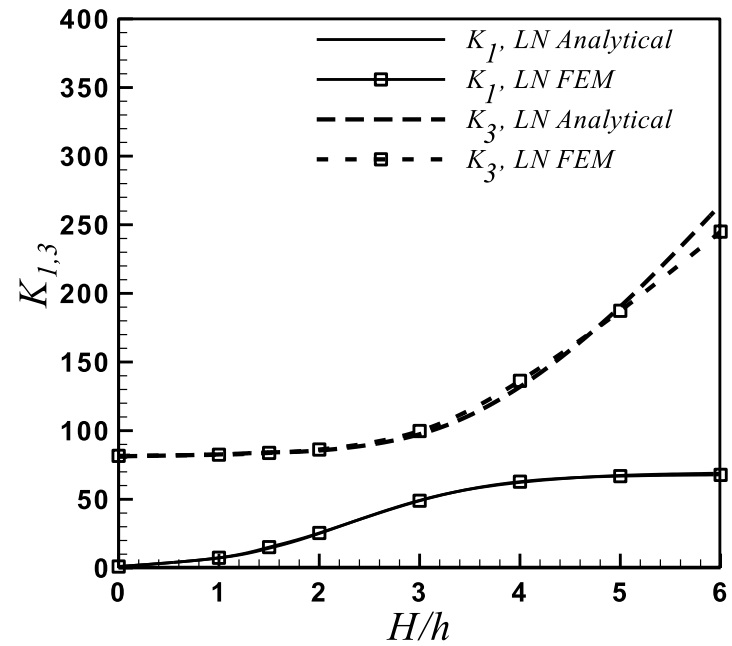

a - Linear frequency analysis

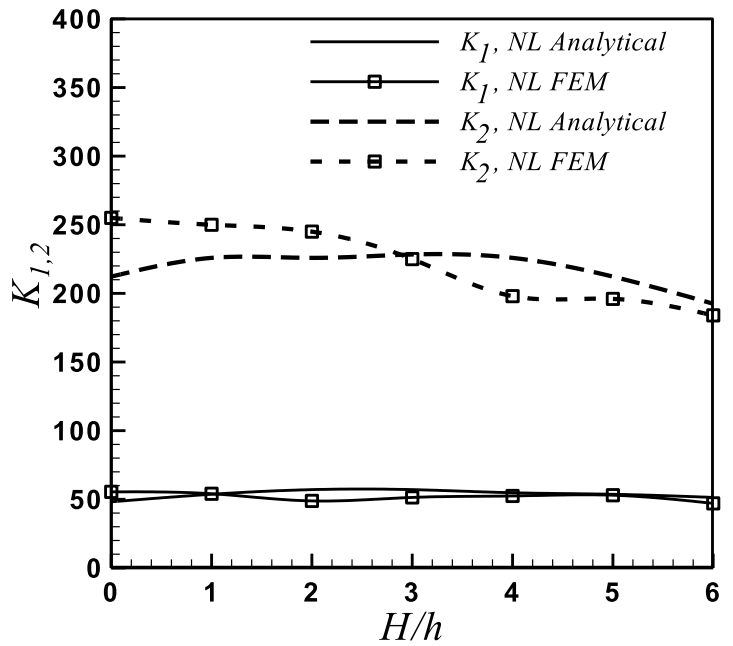

b - Non-linear frequency analysis

Fig. 16 Comparison of changes in the linear and nonlinear frequencies of the curved panel in terms of curvature change

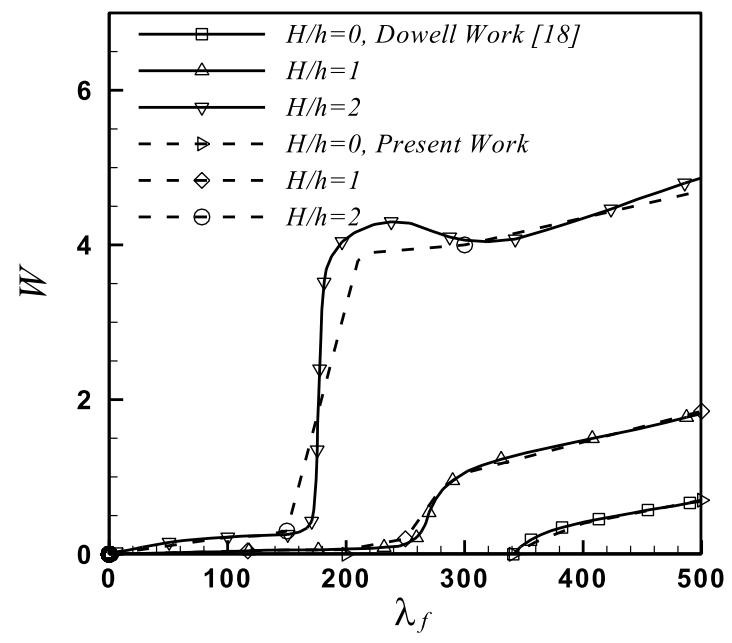

a - Curved plate, $H / h=0,1,2$

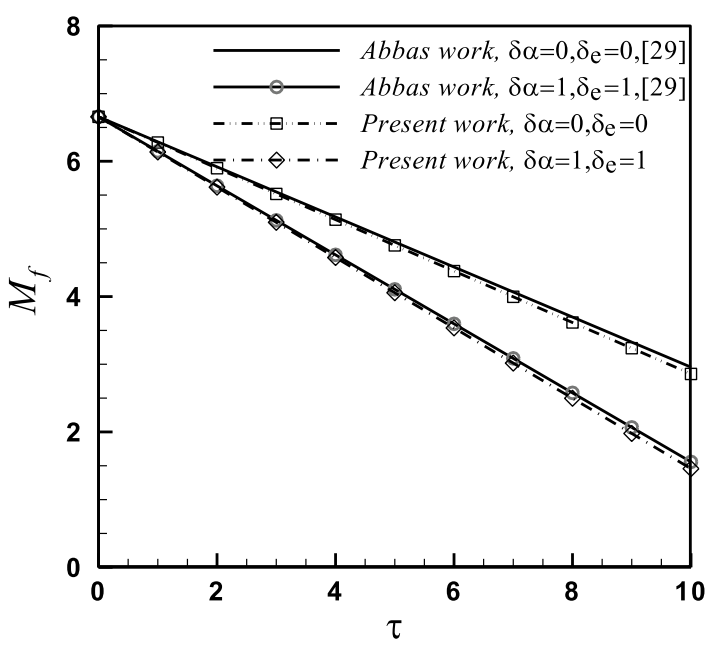

b - Curved plate, $H / h=1$

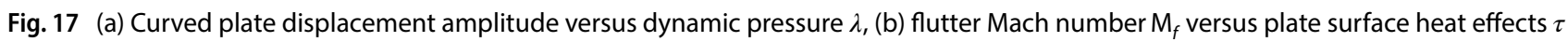

present study is compared with the research of Dowell [18] and Anderson [13]. In Fig. 18b, the trend of nondimensional dynamic pressure changes for the panel with constant boundary conditions for the experimental and analytical model is the same in terms of quality as increasing the curvature of the panel, but differs slightly in value due to differences in laboratory and 3D modeling of the experimental model.

\section{Discussion}

The main topic of discussion in this paper includes the analysis of the nonlinear flutter and post-flutter behavior of the homogeneous metal shell or curved plate with the assumption of nonlinear aerodynamic and nonlinear structure model. The point to consider in terms of our approach 


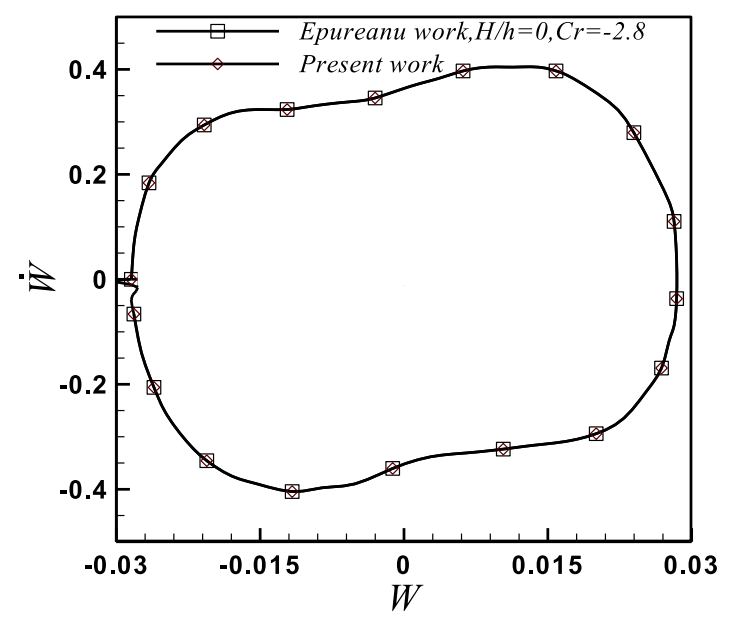

a - compression load, $C_{r}=-2.8[38]$

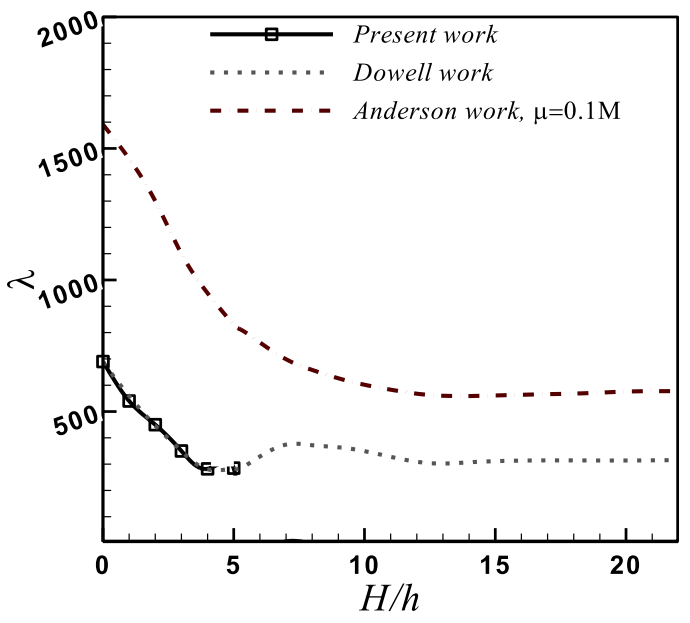

b $-\lambda V s . H / h[13,18]$

Fig. 18 The LCO with compression load effect on nonlinear panel and dynamic pressure versus curvature ratio in supersonic flow regime

in this paper is to analyze the nonlinear behavior of the structure in terms of frequency behavior. In fact, relative awareness of the nonlinear behavior of the structure is obtained, and then the aerodynamic effect is applied to the plate and the flutter behavior is investigated.

The innovation of the article includes the analysis of nonlinear flutter of curved plate with the effect of thermal and mechanical loads in combination with the assumption of aerodynamics of the $3^{\text {rd }}$ order piston theory. A comprehensive review of the above combination has not yet been made.

In the latest paper presented by Amirzadegan et al. [38], flutter study was performed with a pre-stress effect for the isotropic plate. In another paper by Joe et al. [39], the panel flutter was examined focusing on thickness changes, boundary conditions, and the ratio of length to width. In another study by Koo et al. [40], panel flutter examined with the effect of heat to determine the hopf-bifurcation. In another paper, Muc et al. [41], Examined the optimization of plate and shell structure under the influence of the flutter phenomenon.

As it turns out, the present study, from another perspective, has a complete analysis of the parameters affecting the plane flutter.

With the use of Gallerkin method, the results are comparable and validated with previous works. From the perspective of thermal load, the curvature effect of the plate, the effect of mechanical load and the frequency of nonlinear structure, comparison has been made.

The results of the panel flutter are well developed and the curvature effect on the flutter behavior can be improved in the small curvature and in the large curve reducing the flutter boundary.

\section{Conclusion}

The supersonic flutter behavior of 2D curved panels was described using Galerkin method. Structural non-linearities were considered according to the developed formulation. Numerical investigation of the aero-thermo-elastic system including curvature ratio, in-plane load, thermal load distribution on panel as a function of length, thickness ratio, temperature dependency of material properties, first- and third-order piston theory as well as structural analysis based on frequency approach were discussed.

The following conclusions can be drawn from this study:

With an increase in panel curvature, the first- and thirdorder linear structural frequencies increase, although the nonlinear frequencies is fairly constant. For the panel with the camber ratio of 1 , as the in-plane compressive load increases in the range $-10<C_{r}<5$, the frequency of nonlinear structure decreases in linear behavior and increases depending on the non-linear effects. For curved panel with the change $-1.2<C_{r}<1.2$ from tension to compression, the LCO changes from periodic to chaotic motion. With an increase in panel camber ratio from 0 to $5, \mathrm{LCO}$ appears for $0<H / h<1$, after which the chaotic motion is noticed for $1.5<H / h<3$ and the LCO bound is seen for the camber ratios of 4 and 5 . Thus, the aeroelastic coupling behavior on the cambered panel is sensitive to panel camber ratio. With increasing panel curvature to 1.5 , the flutter Mach number of non-linear structure increases; whereas for the 
curvature of 1.5 to 5 , the flutter Mach number decreases. The flutter Mach number with aerodynamic theory of PTA3 decreases compared with that of PTA1.

With an increase in panel curvature from 0 to 3, the panel dynamic displacement increases to the maximum point, while for larger values, the dynamic displacement decreases or remains the same. With the use of PTA3, the panel dynamic displacement slightly decreases. With increase in-plane and thermal load on panel, the flutter frequency and flutter dynamic pressure decrease continuously. Run time is really different between PTA1 and PTA3. Because of more nonlinear term in PTA3, the run time increase progressively.

For future precise work, more detail and accurate flow regime must be replaced instead of PTA theory. Also, 3D panel should be used with experimental data comparison.

\section{Compliance with ethical standards}

Conflict of interest The authors declare that they have no conflict of interest.

\section{References}

1. Jordan PF (1956) The physical nature of panel flutter. Aeronaut Digest 72:34-38

2. Dowell EH, Voss HM (1965) Experimental and theoretical panel flutter studies in the Mach Number range 1.0 to 5.0. AIAA J 3(12):2292-2304

3. Dugundji J (1966) Theorwtical considerations of panel flutter at high supersonic Mach numbers. AIAA J 4:1257-1266

4. Kuo Ch, Morino L, Dugundji J (1972) Perturbation and harmonic balance methods for non-linear panel flutter problem. AIAA J 10:1479-1484

5. Dowell EH, llgamov M (1988) Studies in nonlinear aeroelasticity. Springer, New York

6. Dorsey JC (2002) Metalic thermal protection system technology development: concepts, requirements and assessment overview. In: AIAA 40th Aerospace Science Meeting, Reno

7. Guo X, Mei C (2006) Application of aeroelastic modes on nonlinear supersonic panel flutter at elevated temperatures. Comput Struct 84:1619-1628

8. Culler AJ, McNamara JJ (2010) Studies on fluid-thermal-structural coupling for aero-thermo-elasticity in hypersonic flow. AIAA J 48:1721-1738

9. Perez R, Wang XQ, Mignolet MP (2011) Nonlinear reduced-order models for thermoelastodynamic response of isotropic and functionally graded panels. AIAA J 49:630-641

10. Visbal MR (2012) On the interaction of an oblique shock with a flexible panel. J Fluids struct 30:219-225

11. Fung YC (1954) The static stability of a two dimensional curved panel in a supersonic flow with an application to panel flutter. J Aeronaut Sci 21:556-565

12. Yates JE, Zeijdel EFE (1959) Flutter of curved panels. Midwest Research Institute

13. Anderson WJ (1962) Experiments on the flutter of flat and slightly curved panels at Mach number 2.81. Air Force Office of Scientific Research, Pasadena, AD0419378
14. Stearman RO, Lock MH, Fung YC (1962) Ames tests on the flutter of cylindrical shells, aeroelasticity and structural dynamics. California Institute of Technology, Pasadena, SM 62-37

15. Bolotin VV (1963) On the density of the distribution of natural frequencies of thin elastic shells. J Appl Math Mech 27:538-543

16. Houbolt JC (1965) A study of several aerothermoelastic problems of aircraft structures in high-speed flight 5

17. Schaeffer HG, Heard WI Jr (1965) Flutter of a flat panel subjected to a non-linear temperature distribution. AIAA J 8:1918-1923

18. Dowell EH (1969) Nonlinear flutter of curved plate, part 1. AIAA J:424-431

19. Ventres CS, Dowell EH (1970) Comparison of theory and experiment for non-linear flutter of loaded plates. AIAA J 8:2022-2030

20. Yang TY, Han AD (1976) Flutter of thermally buckled finite element panels. AIAA J 4:975-977

21. Xue DY, Mei C (1993) Finite element non-linear panel with arbitrary temperatures in supersonic flow. AIAA J 31:154-162

22. Bein T, Friedmann PP, Zhong X (1993) Hypersonic flutter of a curved shallow panel with aerodynamic heating. AIAA J

23. Zhou RC, Xue DY, Mei C (1994) Finite element time domain modal formulation for non-linear flutter of composite panels. AIAA J 32:2044-2052

24. Librescu L, Nemeth MP, Starnes JH (1996) Vibration of geometrically imperfect panels subjected to thermal and mechanical loads. J Spacecr Rockets 33:285-291

25. Librescu L, Lin W (1997) Vibration of thermomechanically loaded flat and curved panels taking into account geometric imperfections and tangential edge restraints. Int J Solids Struct 34:2161-2181

26. Gee DJ, Sipcic SR (1999) Coupled thermal model for non-linear panel flutter. AIAA J 37:624-649

27. Librescu L, Marzocca P, Silva WA (2004) Linear/non-linear supersonic panel flutter in a high-temperature field. J. Aircraft 41:918-924

28. Yang J, Shen HS (2003)."onlinear bending analysis of shear deformable functionally graded plates subjected to thermomechanical loads under various boundary conditions. Compos B: Eng 34(2)

29. Abbas LK, Rui X, Marzocca P, Abdalla M, De Breuker R (2011) A parametric study on supersonic/hypersonic flutter behavior of aero-thermo-elastic geometrically imperfect curved skin panel. Acta Mech 222:41-57

30. Yang C, Wan Z (2012) Aerothermal-aeroelastic two-way coupling method for hypersonic curved panel flutter. Sci China Technol Sci 55:831-840

31. Ghoman SS, Azzouz MS (2012) Supersonic aerothermoelastic nonlinear flutter study of curved panels: time domain. J Aircraft 49(4):1178-1183

32. Shahverdi $H$, Khalafi V (2016) Bifurcation analysis of FG curved panels under simultaneous aerodynamic and thermal loads in hypersonic flow. Compos Struct 146:84-94

33. Amirzadegan S, Dowell EH (2019) Nonlinear limit cycle oscillation and flutter analysis of clamped curved plates. J Aircraft 1-9

34. Reddy JN (2003) Mechanics of laminated composite plates and shells. Theory and analaysis, 2nd edn. CRC Press, Boca Raton

35. Miller BA, Mcnamara JJ, Spottswood SM, Culler AJ (2011) The impact of flow induced loads on snap-through behavior of acoustically excited, thermally buckled panels. J Sound Vib 330:5736-5752

36. Dowell EH (1975) Aeroelasticity of plates and shells. Noordhof

37. Epureanu BI, Tang LS, Paidoussis MP (2004) Coherent structures and their influence on the dynamics of aeroelastic panels. Int J Non-Linear Mech 39:977-991

38. Amirzadegan S, Mousavi Safavi SM, Jafarzade A (2019) Supersonic panel flutter analysis assuming effects of initial structural stresses. J Inst Eng India Ser C 100:833-839 
39. Zhou X, Wang L, Jiang J, Su Z (2019) Hypersonic aeroelastic response of elastic boundary panel based on a modified fourier series method. Int J Aerosp Eng. 13, Article ID 5164026

40. Cao L, Yao G (2019) Hopf bifurcation of heated panels flutter in supersonic flow. Mathematics 7(9):787
41. Muc A, Flis J, Augustyn M (2019) Optimal design of plated/shell structures under flutter constraints. Materials 12(24):4215

Publisher's Note Springer Nature remains neutral with regard to jurisdictional claims in published maps and institutional affiliations. 